\title{
Improving the interpretation of electrocardiographs in an accident and emergency department
}

\author{
T White, P Woodmansey, DG Ferguson, KS Channer
}

\begin{abstract}
Summary
In this study we have examined the ability of senior house officers in the Accident and Emergency (A\&E) Department to interpret electrocardiographs (ECGs) and tested the value of a single seminar and guidelines on interpretation. We prospectively audited ECG interpretation taken from notes over a two-month period and repeated this audit following a single seminar by a consultant cardiologist and after the issue of guidelines. $A$ formal test of interpretation of a set of 20 ECGs by senior house officers in A\&E was also carried out. 245 case notes were reviewed and in one third the ECG was interpreted incorrectly by senior house officers in $A \& E$ but incorrect clinical management followed in only $3.2 \%$ of cases. Following the intervention, 242 case notes were reviewed and serious misinterpretations were halved as was the number of patients mismanaged as a result $(1.7 \%)$. In conclusion, formal training in ECG interpretation can reduce serious errors.
\end{abstract}

Keywords: electrocardiograph interpretation, accident and emergency department, medical education

\section{Introduction}

The responsibility for the decision to admit or discharge patients from the Accident \& Emergency (A\&E) department rests mainly with the A\&E senior house officers (SHOs). These are the 'gate-keepers' to the hospital system, granting or denying entry and influencing management by the decision of which area of the hospital to admit to. They are junior medical staff with limited experience of acute medicine, yet are often faced with patients with acute medical conditions like myocardial infarction, where rapid diagnosis is essential for optimum management. Difficult diagnostic decisions must be taken quickly and often in an atmosphere of high pressure. In the assessment of patients with suspected myocardial infarction, the electrocardiograph (ECG) is the most commonly performed investigation. It is used not only to help with diagnosis but also to decide who should receive thrombolysis. ${ }^{1}$ However, the ECG has limitations and at its most informative can only be as accurate as the clinician who interprets it. Incautious or inaccurate interpretation may have important consequences, especially in the A\&E department where triage decisions are made and events can develop rapidly. The ability of the SHO to interpret the ECG and evaluate and act on the information gleaned in an appropriate manner is important for good clinical care.

Previous studies have examined the management of patients admitted to the A\&E department with chest pain. Emerson and colleagues ${ }^{2}$ showed that $12 \%$ of patients were discharged in error and $16 \%$ were admitted unnecessarily. When the interpretation of the ECG was reviewed it was found that misinterpretation of this was responsible for $4 \%$ of the inappropriate discharges and $1 \%$ of unnecessary admissions. In a similar study McCallion and colleagues ${ }^{3}$ reviewed the accuracy of ECG interpretation by SHOs from 279 patients with chest pain and compared this to the interpretation by a consultant cardiologist. They found that the SHOs correctly identified $90 \%$ of normal and $57 \%$ of abnormal ECGs. However, despite misinterpretation of $43 \%$ of abnormal ECGs, $97 \%$ of patients were managed correctly. Morrisson and $\mathrm{Swann}^{4}$ investigated interpretation of ECGs by A\&E SHOs and found that $20 \%$ of the ECGs were interpreted incorrectly when compared to a cardiologists' report but only $4.4 \%$ were considered to be clinically important. In an American study, Westdrop et $a l \bar{l}$ investigated the interpretation of 760 ECGs and identified 143 abnormal recordings from patients discharged from an Emergency room. In 83/143 (58\%) the cardiologist disagreed with the emergency room physician but in only $25(18 \%)$ was the discrepancy thought to be clinically significant. Thus, although the ECG may be misinterpreted on a technical level, inappropriate management results in only a minority of cases. In our study we have evaluated an intervention to assess the benefits for interpretation of ECGs by SHOs in an A\&E department.

\section{Study 1: Audit of the interpretation of ECGs in A\&E}

\section{METHODOLOGY}

The study was carried out prospectively in two phases between April and July 1992. All patients presenting to the $A \& E$ department at 
the Royal Hallamshire Hospital during the two periods of study and in whom an ECG was performed were included in the study, regardless of clinical presentation. Duplicate copies of all ECGs were made. The original ECGs were interpreted by the medical staff and attached to the patients A\&E notes. Eleven SHOs were working in the $A \& E$ department at the time in a complex shift system. They were warned of the nature of the study and were asked to ensure that they recorded their interpretation of all ECGs in full on the A\&E notes. They were given no other information or feedback during the course of the study.

Duplicate ECGs were reported by an experienced registrar in cardiology who was blinded to the clinical presentation of the patient, the SHO's interpretation and the subsequent management of the patient. In cases of doubt the ECGs were submitted to a consultant cardiologist for opinion, as were one in $\mathbf{1 0}$ for confirmation of the cardiology registrar's interpretation (quality control).

The A\&E notes referring to the ECGs were drawn from medical records and the SHO's interpretation recorded verbatim. Where sets of notes could not be located and when there was no report of the SHO's interpretation of the ECG in the notes, this was recorded. The interpretations by $\mathrm{SHO}$ and cardiology registrar were compared and the accuracy of the SHO's interpretation was scored on a scale from 1 to 4 using the grading system shown in table 1. Misinterpretations included the failure to detect an abnormality (false negative) and the reporting of an abnormality when none existed (false positive). Where the assessor could not confidently give an opinion the case was submitted to the consultant cardiologist for a decision. Where there was significant disagreement between the opinion of the cardiology registrar and the A\&E SHO (category 4) the case was reviewed by the consultant cardiologist. In these cases the A\&E notes were made available so that the ECG diagnosis and subsequent management could be assessed in the context of the history and examination. The consultant did not appraise the results of special investigations or any other information not available to the SHO during the stay of the patient in the $A \& E$ department. In the light of this information the ideal management of the patient was determined. This was subsequently compared to the actual management decision by the SHO, for example to admit to a ward, admit to the coronary care unit, refer to the resident medical officer or discharge the patient. The accuracy of the $A \& E$ officer's management decisions were then graded according to the system shown in table 2 . In cases where interpretation of the ECG was incorrect the areas most commonly misinterpreted were identified.

\section{Intervention}

Following the initial prospective audit the effect of an intervention was studied. A presentation by the consultant cardiologist was given to all the A\&E SHOs in which the principles of ECG interpretation were summarised. The ECG manifestations and clinical importance of the most common pathologies with ECG correlates were described. In addition the SHOs were given a set of rules for interpretation which were fixed to their $A \& E$ protocol handbooks for ease of reference.

After an interval of four weeks, in order to avoid the effects of short-term memory retention, the prospective audit of ECG interpretation described above was repeated.

\section{Study 2: Formal testing of the interpreta- tion of ECGs by SHOs in A\&E}

\section{METHODOLOGY}

In order to test the ability of the SHOs to interpret ECGs formally, they were given a set of 20 ECGs which had been recorded from patients attending the $A \& E$ department in the preceding month. The doctors were asked to describe the rate, rhythm and axis and to report any abnormalities seen on a standardised answer paper. Their interpretation was compared to the consultant cardiologist's interpretation of the ECG using the same scoring system as in table 1. The ECGs were selected to represent the spectrum of abnormalities seen in the $A \& E$ department and included normal and abnormal recordings. Following the ECG intepretation seminar, a further $20 \mathrm{ECG}$ tests were repeated. In order to ensure the comparability of the tests before and after intervention two sets of ECGs were used. Half the SHOs were given one set of

Table 1 Classification of grades of agreement

\begin{tabular}{|c|c|c|}
\hline Grade & Definition & Criteria \\
\hline 1 & Complete concordance & Accurate interpretation of the ECG \\
\hline 2 & Minor disagreement & $\begin{array}{l}\text { First degree heart block, ventricular ectopic beats, sinus tachycardia, } \\
\text { sinus bradycardia, minor non-specific } T \text { wave changes }\end{array}$ \\
\hline 3 & Disagreement & $\begin{array}{l}\text { Left ventricular hypertrophy, right or left axis deviation, bundle } \\
\text { branch block, slow atrial fibrillation, isolated } T \text {-wave inversion or } \\
\text { non-specific ST-segment depression, identification of } Q \text { wave in a lead } \\
\text { in which it is not significant, Wolff-Parkinson-White syndrome }\end{array}$ \\
\hline 4 & $\begin{array}{l}\text { Significant disagreement, } \\
\text { potentially affecting } \\
\text { immediate management }\end{array}$ & $\begin{array}{l}\text { ST-segment depression or elevation, widespread T-wave changes or } \\
\text { pathological Q-wave development, fast atrial fibrillation or other dysr- } \\
\text { hythmia }\end{array}$ \\
\hline
\end{tabular}


Table 2 Management decision criteria in cases of significant disagreement on ECG interpretation

\begin{tabular}{ll}
\hline Grade & Description \\
\hline 4a & $\begin{array}{l}\text { Patient correctly managed despite SHO having significantly } \\
\text { misinterpreted the ECG }\end{array}$ \\
4b & $\begin{array}{l}\text { Patient admitted unnecessarily, substantially as a consequence of } \\
\text { incorrect ECG interpretation }\end{array}$ \\
4c & $\begin{array}{l}\text { Patient discharged in error, substantially as a consequence of } \\
\text { incorrect ECG interpretation }\end{array}$ \\
\hline
\end{tabular}

ECGs (set A), while the other half were given a different set (set B). Following intervention the group given set $\mathrm{A}$ were given set $\mathrm{B}$ and vice versa. The two sets were matched for difficulty as far as possible.

\section{STATISTICAL METHODS}

Two-tailed Chi-square analysis was used to test for differences between the distribution in the grades of agreement before and after intervention.

\section{Results}

\section{STUDY 1}

Before intervention a total of 349 ECGs were obtained. Of these, there were no patient details for nine and 15 referred to patients whose notes could not be located. There were a further 80 in which were was no ECG interpretation recorded in the notes. All these were excluded

Table 3 Audit of A\&E SHO's interpretation of electrocardiographs. Numbers $(\%)$ in each grade of agreement before and after intervention

\begin{tabular}{llll}
\hline Grade & Definition & $\begin{array}{l}\text { Before } \\
\text { intervention }\end{array}$ & $\begin{array}{l}\text { After } \\
\text { intervention }(\%)\end{array}$ \\
\hline 1 & Complete concordance & $114(46)$ & $109(45)$ \\
2 & Minor disagreement & $52(21)$ & $51(21)$ \\
3 & Disagreement & $28(11)$ & $57(24)$ \\
4 & Significant disagreement: & $43(18)$ & $21(9)$ \\
& (a) correct management & $1(0.4)$ & 0 \\
& (b) unnecessary admission & $7(2.9)$ & $4(1.7)$ \\
& (c) inappropriate discharge & & 242 \\
\hline
\end{tabular}

Table 4 Number and description of interpretation errors (grade 3) before and after intervention

\begin{tabular}{|c|c|c|c|c|}
\hline \multirow[b]{2}{*}{ Aspect misinterpreted } & \multicolumn{2}{|c|}{ Before intervention } & \multicolumn{2}{|c|}{ After intervention } \\
\hline & False +ve & False-ve & False + ve & False-ve \\
\hline Left ventricular hypertrophy & 1 & 6 & 0 & 15 \\
\hline Bundle branch block & 0 & 2 & 1 & 4 \\
\hline Slow atrial fibrillation & 1 & 1 & 1 & 3 \\
\hline Isolated $\mathrm{T}$-wave change & 3 & 4 & 7 & 9 \\
\hline Axis deviation & 3 & 7 & 6 & 12 \\
\hline Pathological Q wave & 7 & 3 & 12 & 4 \\
\hline Pace-maker rhythm & 0 & 0 & 0 & 1 \\
\hline Wolff-Parkinson-White & 0 & 0 & 0 & 1 \\
\hline Total & 38 & & 76 & \\
\hline
\end{tabular}

leaving 245 ECGs which were used in the initial evaluation. The levels of accuracy found are shown in table 3 . A total of $114(46 \%)$ ECGs were interpreted accurately (grade 1) with a further $52(21 \%)$ rates as having only minor errors (grade 2). Thus, the intepretation of a total of $166(67 \%)$ ECGs was judged to have been substantially correct. In $28(11 \%)$ ECGs at least one important error of interpretation was identified (grade 3) and a further $51(21 \%)$ were misinterpreted to a degree which would potentially affect immediate management (grade 4). Of grade 4 errors, in $43(84 \%$ ) correct management followed despite the ECG error. Of the remainder, seven $(14 \%)$ were discharged in error and one $(2 \%)$ was admitted unnecessarily. Overall, eight out of $245(3.3 \%)$ patients were judged to have been mismanaged as a result of misinterpretation of the ECG.

\section{Aspects of the ECG misinterpreted}

The cases in which the ECGs were misinterpreted (grade $3 \& 4$ ) were further analysed. The results are shown in tables 4 and 5 . The most common grade 3 errors of intepretation were the inability to recognise left ventricular hypertrophy, abnormal cardiac axis, and inappropriate identification of $Q$ waves where these were not significant. Common grade 4 errors included failure to recognise old myocardial infarction and to see changes in ST segments.

\section{Effect of intervention}

After the intervention, 342 ECGs were obtained, of which one had no patient details and 16 referred to patients whose notes could not be located. In 83 cases there was no record of the SHO's intepretation in the notes and these ECGs were excluded leaving 242 ECGs. Of these $109(45 \%)$ were correctly interpreted and $51(21 \%)$ had only minor errors (table 3 ); $57(24 \%)$ were misinterpreted (grade 3$)$ and a further $25(10 \%)$ were misinterpreted to a degree where it could alter patient management (grade 4). Of those cases graded as 4, $21(84 \%)$ were managed correctly and four $(16 \%)$ were discharged in error. No patient was considered to have been admitted unnecessarily. The distribution is similar to that seen before the intervention. The proportion of ECGs in grades $1 \& 2$ were the same before and after intervention, but the proportion of ECGs in grade 4 was halved after intervention $\left(\chi^{2}=18.9, \mathrm{p}<0.005\right)$. The nature of misinterpretation is shown in tables $4 \& 5$. Following the intervention, the number of grade 3 technical errors of interpretation increased but the number of serious grade 4 errors fell significantly $\left(\chi^{2}=28.9 ; \mathrm{p}<0.0001\right)$.

\section{STUDY 2}

The accuracy of the SHO's interpretation of ECGs on formal testing before and after the intervention is shown in table 6 . Within the group $111(56 \%)$ of the ECGs were graded 1 or 2 before the intervention and this rose to $64 \%$ after the intervention. A corresponding decrease was seen in grade 4 errors from 41 $(21 \%)$ to $35(18 \%)$. However, these changes were not significant $\left(\chi^{2}=3.0, \mathrm{p}=\mathrm{ns}\right)$. 
Table 5 Number and description of interpretation errors (grade 4) before and after intervention

\begin{tabular}{|c|c|c|c|c|}
\hline \multirow[b]{2}{*}{ Aspect misinterpreted } & \multicolumn{2}{|c|}{ Before intervention } & \multicolumn{2}{|c|}{ After intervention } \\
\hline & False+ve & False-ve & False+ve & False-ve \\
\hline Acute infarct & 0 & 3 & 0 & 2 \\
\hline ST-segment elevation & 6 & 3 & 3 & 4 \\
\hline ST-segment depression & 4 & 15 & 1 & 6 \\
\hline Widespread $\mathrm{T}$-wave inversion & 3 & 9 & 1 & 8 \\
\hline Fast atrial fibrillation & 0 & 7 & 0 & 0 \\
\hline Posterior infarct & 0 & 2 & 0 & 1 \\
\hline Old infarct & 9 & 7 & 3 & 4 \\
\hline Heart block & 1 & 2 & 0 & 0 \\
\hline Incorrect recording & 0 & 1 & 0 & 1 \\
\hline Paced rhythm & 0 & 1 & 0 & 0 \\
\hline Total & \multicolumn{2}{|l|}{74} & \multicolumn{2}{|l|}{33} \\
\hline
\end{tabular}

Table 6 Formal test of SHOs in A\&E to interpret ECGs. Number $(\%)$ and grade of agreement

\begin{tabular}{llll}
\hline Grade & Definition & $\begin{array}{l}\text { Before } \\
\text { intervention }\end{array}$ & $\begin{array}{l}\text { After } \\
\text { intervention }(\%)\end{array}$ \\
\hline 1 & Complete concordance & $86(43)$ & $102(51)$ \\
2 & Minor disagreement & $25(12.5)$ & $25(12.5)$ \\
3 & Disagreement & $48(24)$ & $38(19)$ \\
4 & Significant disagreement & $41(20.5)$ & $35(17.5)$ \\
\hline
\end{tabular}

\section{Discussion}

Our results show that two-thirds $(67 \%)$ of ECGs were interpreted correctly by SHOs in $\mathrm{A} \& \mathrm{E}$, whilst one-third were misinterpreted; $21 \%$ being misinterpreted to a degree where immediate management might have been affected (grade 4 ), although in fact only $3 \%$ of cases were managed incorrectly as a result. This is very similar to the findings of $\mathrm{McCal}$ lion $^{3}$ and Westdrop ${ }^{5}$ who showed that $27 \%$ and $18 \%$ of ECGs from patients with chest pain, respectively, were interpreted incorrectly. McCallion also reported an inappropriate discharge rate of $2.5 \%$ for patients with chest pain, which is similar to our own finding of $2.8 \%$. These authors also studied the nature of the misinterpretations found and reported that a large proportion of false positive interpretations cited an inferior infarction which again is similar to our findings. We found a general over-interpretation of the importance of $Q$ waves in the inferior leads, especially lead III. By comparison, emergency room doctors in the US apparently under-report infarction and ischaemia. ${ }^{5}$ Similarly, Emerson and colleagues ${ }^{2}$ reported on the management of patients with chest pain presenting to the $A \& E$ department and found a major misinterpretation of the ECG resulted in clinical mismanagement in $1.5 \%$ of cases.

1 Channer KS. Deciding who should have thrombolysis. $B M \mathcal{J}$ 1993; 307: 1146

2 Emerson PA, Russell NJ, Wyatt J, et al. An audit of doctors management of patients with chest pain in the accident and emergency department. $Q \mathcal{F}$ Med 1989; 263: 213-20.

3 McCallion WA, Templeton PA, McKinney LA, Higginson JDS. Missed myocardial ischaemia in the accident and emergency department: ECG a need for audit. Arch Emerg Med 1991; 8: 102-7.

4 Morrison WG, Swann IJ. Electrocardiograph interpretation by junior doctors. Arch Emerg Med 1990; 7: 108-10.
Following the intervention, the number of clinically important errors was halved, as was the rate of inappropriate discharge. No patients were advised admission unnecessarily. However, although the number of grade 4 errors fell, the overall proportion of patients in whom the ECG was incorrectly interpreted remained the same (grades $3 \& 4$ combined). Thus, although significant mistakes were reduced, mistakes not affecting patient management increased proportionally. Our results show that the type of interpretation errors following intervention was similar to that seen before intervention, occurring in all aspects of ECG interpretation although the frequency of serious errors was reduced. Thus, the change has not been the result of an increase or decrease in the error rate of interpretation of any particular part of the ECG. We believe that the teaching produced a reduction in serious errors but exposed an underlying misunderstanding of less serious and more technical aspects of ECG reporting.

\section{Can interpretation skills be improved?}

Gjorup et $a l^{t}$ studied the effect of a more detailed training programme on ECG interpretation ( $8 \mathrm{~h}$ over 14 days) for junior hospital doctors. They found no evidence of any improvement in agreement between doctors on interpretation but did not evaluate clinical decision making. Our data show that the number of serious errors of ECG interpretation can be significantly reduced by simple interventions. It is possible that factors other than the ECG seminar and the provision of guidelines for ECG interpretation contributed to the observed reduction. In particular, SHOs had two months more experience. However, we have tested registrars in ECG interpretation with the ECG test used in this study. They performed no better than SHOs in A\&E and considerably worse than our coronary care unit nurses and cardiographers. This suggests that experience alone is not enough and we should recommend formal ECG training for junior doctors. Do doctors need to be able to interpret ECGs since many modern ECG recorders offer a computer-based interpretation? Willems et $a l$ compared the ability of nine computer programs with that of eight cardiologists to diagnose certain ECG abnormalities (excluding ischaemia). They showed that the computers were significantly inferior to the trained doctors, and concluded that 'review of computerised ECG reports by a physician is therefore still essential'. In conclusion, formal training in ECG interpretation is an important part of postgraduate education in medicine.

5 Westdrop EJ, Gratton MC, Watson WA. Emergency department interpretation of electrocardiograms. Ann Emerg Med 1992; 21: 541-4.

6 Gjorg M, Kelbaek H, Nielsen D, Kreiner S, Godfriedsen J. Interpretation of the electrocardiogram in suspected myocardial infarction: a randomised controlled study of the effect of a training programme to reduce inter-observer variation. F Intern Med 1992; 231: 407-12.

7 Willems JL, Abreu-Lima C, Arnaud P, et al. The diagnostic performance of computer programs for the interpretation of electrocardiograms. N Engl f Med 1991; 325: 1767-73. 